This is an Open Access article distributed under the terms of the Creative Commons Attribution-Noncommercial License, which permits unrestricted use, distribution, and reproduction in any noncommercial medium, provided the original work is properly cited.

\title{
PROTOPLANETARY GAS DISKS IN THE FAR INFRARED
}

\author{
Javier R. Goicoechea ${ }^{1}$ and Bruce Swinyard ${ }^{2}$ \\ on behalf of the SPICA/SAFARI science team \\ ${ }^{1}$ Centro de Astrobiología, CSIC-INTA, Madrid, Spain \\ ${ }^{2}$ Rutherford Appleton Laboratory, Chilton, Didcot, Oxfordshire, UK
}

\section{ABSTRACT}

The physical and chemical conditions in young protoplanetary disks set the boundary conditions for planet formation. Although the dust in disks is relatively easily detected as a far-IR photometric "excess" over the expected photospheric emission, much less is known about the gas phase. It seems clear that an abrupt transition from massive optically thick disks (gas-rich structures where only $\sim 1 \%$ of the total mass is in the form of dust) to tenuous debris disks almost devoid of gas occurs at $\sim 10^{7}$ years, by which time the majority of at least the giant planets must have formed. Indeed, these planets are largely gaseous and thus they must assemble before the gas disk dissipates. Spectroscopic studies of the disk gas content at different evolutive stages are thus critical. Far-IR water vapour lines and atomic fine structure lines from abundant gas reservoirs (e.g., [O I] $63 \mu \mathrm{m},[\mathrm{S} \mathrm{I}] 56 \mu \mathrm{m},[\mathrm{Si} \mathrm{II}] 34 \mu \mathrm{m})$ are robust tracers of the gas in disks. Spectrometers on board Herschel will detect some of these lines toward the closest, youngest and more massive protoplanetary disks. However, according to models, Herschel will not reach the required sensitivity to (1) detect the gas residual in more evolved and tenuous transitional disks that are potentially forming planets and (2) detect the gas emission from less massive protoplanetary disks around the most numerous stars in the Galaxy (M-type and cooler dwarfs). Both are unique goals for SPICA/SAFARI. Besides, SAFARI will be able to detect the far-IR modes of water ice at $\sim 44$ and $\sim 62 \mu \mathrm{m}$, and thus allow water ice to be observed in many protoplanetary systems and fully explore its impact on planetary formation and evolution.

Key words: stars: planetary systems: formation - protoplanetary disks - infrared: stars - Missions: SPICA

\section{INTRODUCTION}

All planets are thought to form in the accretion disks that develop during the collapse and infall of massive dusty and molecular cocoons $(\geq 10,000 \mathrm{AU})$ where stars are born. However, we still have a very incomplete understanding of the physical and chemical conditions in such circumstellar disks, how they evolve when dusty bodies grow and collide, which is their mineral content, how they clear as a function of time and, ultimately, how planets as diverse as the Earth or hot-Jupiters form around different types of stars and at different places of the galaxy.

Circumstellar disks are divided into 3 classes according to their evolutionary stage. Primordial protoplanetary disks, which are rich in atomic and molecular gas, and are composed of relatively unprocessed interstellar material left over from the star formation process. Such young disks are very optically thick in dust, with high radial midplane optical depths in the visual $\left(\tau_{V}>>1\right)$. These protoplanetary disks start to become optically thin $\left(\tau_{V} \simeq 1\right)$ in a few million years after formation (Haisch et al., 2001) and evolve into transitional disks as their inner regions begin to clear at $\geq 10 \mathrm{Myr}$. This is the critical intermediate stage when planetary formation is believed to take place, with dust particles colliding and growing to form larger bodies reducing the disk opacity. Spitzer has shown that their outer regions (beyond $\sim 10-20 \mathrm{AU}$ ) can remain intact for longer, and thus residual gas can exist in the disk and play an important role in its evolution. In spite of its importance, the residual gas content in transitional disks at the first stages of planet formation is very poorly constrained, and many clues on these early stages of planetary formation can be provided by spectral line observations. Disks with ages above $\geq 10$ Myr are thought to be practically devoid of gas (Duvert et al., 2000) and the dust in these older debris disks is generally not primordial but continuously generated "debris" from planetesimal and rocky body collisions. The smallest dust grains have, at this stage, either been dispersed or have coagulated into larger grains and the disk becomes very optically thin $\left(\tau_{V}<<1\right)$. Debris disks are thus younger and more massive analogs of our own asteroid (hot inner disk, $T_{d} \simeq 200 \mathrm{~K}$ ) and Kuiper belts (cool outer disk, $T_{d} \simeq 60 \mathrm{~K}$ ) so their study is vital to place the Solar System in a broader context (see more detailed contributions in these proceedings).

\section{The GAS CONTENT IN PLANET FORMING DISKS}

The physical and chemical conditions in young protoplanetary disks (primordial and transitional) set the boundary conditions for planet formation and an understanding of the formation and evolution of such disks will finally link star formation and planetary science. Planets are believed to form within the inner $\sim 20$ AU of transitional protoplanetary disks which emit most of their energy in the 


\section{"Transitional" protoplanetary disks ( 10Myr): spectral diagnostics for SPICA/SAFARI}

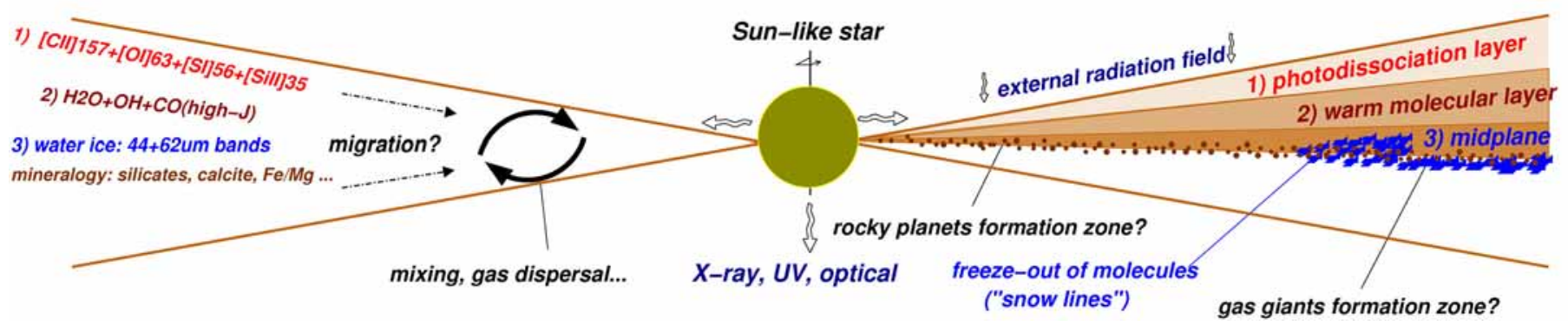

Figure 1. Diagram showing where radiation arises from a transitional protoplanetary disk (at the time when planets are thought to assemble) and why the far-IR (i.e., SPICA/SAFARI) and the millimetre (e.g., ALMA) together are essential to understanding the full picture of planetary formation and the primordial chemistry that may eventually lead to the emergence of life.

mid- and far-IR region (Boss, 2008). Although the dust is relatively easily detected by photometric observations in the far-IR range, very little is known about the gas phase. It seems clear that an abrupt transition from massive optically thick disks to tenuous debris disks occurs at $\geq 10 \mathrm{Myr}$ (Meyer et al., 2008), by which time the majority of at least the giant gaseous planets (e.g., "Jupiters" and "hot Jupiters") must have formed. The very fact that these planets are largely gaseous means they must be formed before the gaseous disk dissipated, making the study of the gas in protoplanetary disks essential to an understanding of how and where they formed. For instance, the recently detected massive "hot Jupiters" orbiting close to the parent star are very unlikely to have formed in situ, but rather they must have migrated inwards from outer disk regions beyond the snow line (see Sect. 3.3). Likewise it seems clear that Neptune and Uranus must have formed in a region closer to the Sun and migrated outwards. The mechanism for either of these scenarios is not certain.

Protoplanetary disk models predict a "flared" structure (see Fig. 1) which allows the disk to capture a significant portion of the stellar UV and X-ray radiation even at large radii (Qi, 2006; Bergin et al., 2007) boosting the dust thermal emission, and the emission of mid- and farIR lines from gas phase ions, atoms and molecules. Theoretical models recognise the importance of these X-UV irradiated surface layers, which support active photochemistry and are responsible for most of the mid- and far-IR line emission. They are, in many ways, similar to the well studied photodissociation regions of the ISM, that show a very rich far-IR spectrum (e.g., Goicoechea et al. 2004; 2009). Fine structure lines of the most abundant elements and metals (O, C, S, Si...) together with the far-IR rotational line emission of light hydrides $\left(\mathrm{H}_{2} \mathrm{O}, \mathrm{OH} \ldots\right)$ are predicted to be the brightest gas cooling lines of the warm disk, especially close to the star (Gorti \& Hollenach 2004, 2008; Woitke et al. 2009a, 2009b; Cernicharo et al. 2009).

Indeed, far-IR [Si II]34, [O I]63,145, [S I] 56 and [C II]158 $\mu \mathrm{m}$ fine structure lines can be brighter than the mid-IR $\mathrm{H}_{2}$ emission, the most abundant gas species in the disk.
By detecting these lines we can directly probe the physical and chemical conditions in disks around a large number of stars at differently evolutionary stages. Herschel can only search for the strongest lines (above a few $10^{-18} \mathrm{~W} \mathrm{~m}^{-2}$ ) toward the brightest, closest and most massive young protoplanetary disks. In fact, only a few disks will be fully surveyed in the far-IR i.e., those with strong dust continuum which unfortunately difficulties the line detection at low spectral resolution. In particular Herschel does not reach the sensitivity to either detect the gas emission in the much more tenuous transitional disks or detect the predicted gas line emission of less massive, and thus more numerous, disks around cooler stars.

\section{SPICA STUDIES OF THE GAS DISPERSAL}

In order to shed some light on the gas dispersal time scales, and thus on the formation of gaseous Jovian-type planets, high sensitivity infrared to submm spectroscopic observations over large statistical stellar samples tracking all relevant disk evolutionary stages and stellar types are clearly needed. To date, studies of the gas content are biased to young and massive protoplanetary disks (probably not the most representative) through observations with ground-based (sub)mm interferometers and optical/nearIR telescopes. Submm observations allows one to trace the outer and cooler disk extending over a few hundred AU (Dutrey et al., 2007). However, in this region most molecular species freeze-out onto dust grain icy mantles and thus the determination of the total gas mass can be uncertain. Recently the very inner regions of protoplanetary disks have been probed by means of optical/IR observations (Najita et al., 2007). Spitzer and ground-based telescopes have just started to show the potential diagnostic power of mid- and far-IR spectroscopy in a few "template" disks. This has allowed the exploration of the gas content and composition at intermediate radii (1-30 AU), i.e., the crucial region for the formation of planets. Surprisingly, recent mid-IR detections toward young disks include atomic lines such as [NeII], [Fe II], etc. (Lahuis et al., 


\section{Expected gas line emission in transitional protoplanetary disks with SPICA/SAFARI}
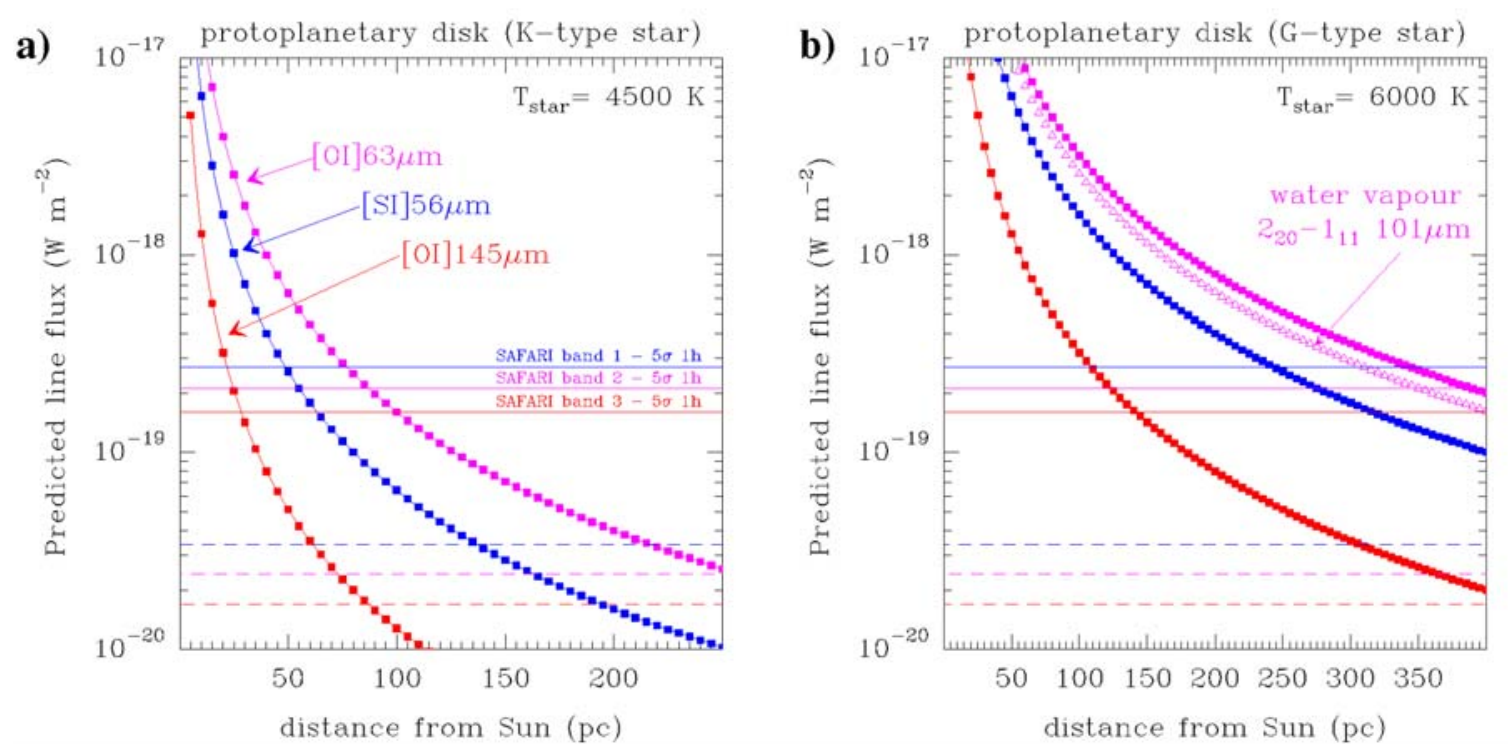

Figure 2. Predicted fluxes of some key far-IR cooling lines in transitional protoplanetary disks ( 10Myr) around (a) a cool K star and (b) a $G$ solar-type as a function of distance. The horizontal lines indicate $5 \sigma-1 \mathrm{hr}$ detection limits of SAFARI. Dashed lines correspond to sensitivities using narrow band filters on a restricted number of wavelengths. The gas mass in the modelled disk is $10^{-2} M_{\text {Jup. }}$ SPICA will have the sensitivity to observe the gas content of disks in the closest star forming regions ( $\sim 150$ pc) at the time planets assemble. Adapted from Gorti 83 Hollenbach (2004) models.

2007), molecular species like $\mathrm{H}_{2}, \mathrm{H}_{2} \mathrm{O}, \mathrm{OH}, \mathrm{HCN}, \mathrm{C}_{2} \mathrm{H}_{2}$ and $\mathrm{CO}_{2}$ (Carr \& Najita, 2008; Salyk et al., 2008), and even more complex organic species such as PAHs (Geers et al., 2006; Habart et al., 2006) that chemical models are now trying to reproduce (e.g., Agúndez et al. 2008). SPICA spectrometers will provide continuous wavelength coverage from $\sim 5$ to $210 \mu \mathrm{m}$ for the first time since the launch of ESA's Infrared Space Observatory (1995).

Molecular hydrogen $\left(\mathrm{H}_{2}\right)$ is the most abundant gas species in a primordial protoplanetary disk $(\sim 90 \%$ of the initial mass). Due to the relatively poor line sensitivity that can be achieved with even the largest ground-based mid-IR telescopes, $\mathrm{H}_{2}$ has been detected only toward a few protoplanetary disks so far (Bitner et al., 2008). JWST will improve this situation enormously and many more disks will have been observed by the time SPICA is active. To complete and complement the JWST capability, SPICA's mid-IR high resolution echelle spectrometer (MIRHES) will be able to detect the brightest $\mathrm{H}_{2}$ line (the $v=0-0 S(1)$ line at $\sim 17 \mu \mathrm{m})$ with $\sim 10 \mathrm{~km} \mathrm{~s}^{-1}$ resolution and much higher sensitivities than those achieved from the ground and with an order of magnitude higher spectral resolution than JWST. Such a high spectral resolution will resolve the gas Keplerian rotation, trace the warm gas in the inner $(<30 \mathrm{AU})$ disk regions and be sensitive to $\mathrm{H}_{2}$ masses of the order of $\sim 0.1 \mathrm{M}_{\text {Earth }}$.

However, given the peculiar excitation conditions of $\mathrm{H}_{2}$ (e.g., in the warm UV illuminated edge of the disk and in the hottest inner regions), the $\mathrm{H}_{2}$ emission may not sample the whole circumstellar disk. Instead, the deuterated isotopologue of molecular hydrogen, $\mathrm{HD}$, although a few thousand times less abundant, can serve as a proxy of $\mathrm{H}_{2}$ column density. The $\mathrm{D} / \mathrm{H}$ abundance ratio of different species can also be used as a diagnostic of the ISM origin of the young disk material. HD has a small permanent dipole moment and its lowest energy rotational lines appear in the range covered by SAFARI (112, 56 and $37 \mu \mathrm{m})$.

\subsection{Line Detectability with SPICA/SAFARI}

At a distance of $\sim 50 \mathrm{pc}$ from the Sun, a given gas line will produce a flux of $\sim 10^{-19}\left(\mathrm{~L}_{\text {line }} / 10^{-8} \mathrm{~L}_{\odot}\right) \mathrm{W} \mathrm{m}^{-2}$ where $\mathrm{L}_{\text {line }}$ is the radiated power in the line expressed in solar luminosities. SAFARI will be able to detect gas lines with luminosities $\geq 10^{-8} \mathrm{~L}_{\odot}$ in nearby, isolated, disks, or $\geq 10^{-7} \mathrm{~L}_{\odot}$ in more distant disks in the closest star forming regions at $\sim 150 \mathrm{pc}$ (see Figure 2). State-of-the-art models of transitional disks around Sun-like stars and with gas masses as low as $\sim 0.001 \mathrm{M}_{\text {Jup }}\left(\sim 25 \mathrm{M}_{\text {moon }}\right)$ predict luminosities around $10^{-8} \mathrm{~L}_{\odot}$ for [O I] $145 \mu \mathrm{m}$, [S I] $56 \mu \mathrm{m}$ and $\mathrm{H}_{2} \mathrm{O}$, OH or CO high- $J$ lines (Gorti \& Hollenbach, 2004). The $[\mathrm{O} \mathrm{I}] 63 \mu \mathrm{m}$ line is expected to be the brightest line $\left(10^{-6}-10^{-7} \mathrm{~L}_{\odot}\right)$ even for modest gas masses and therefore it is one of the most robust gas tracers. The exciting prospect of detecting this small amount of gas in a statistically significant sample of disks, e.g., toward the 6 closest $(\leq 150 \mathrm{pc})$ young stellar clusters with ages of $\simeq 1-30 \mathrm{Myr}$ : 
Resolving the "snow line" in nearby Vega-like disks + detailed mineralogy of hundred's of debris disks

a)

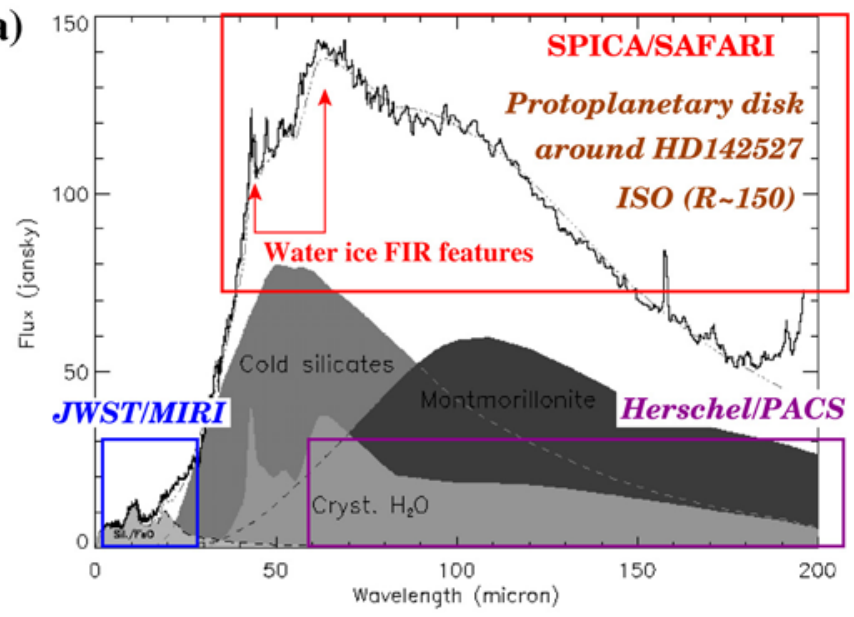

b)

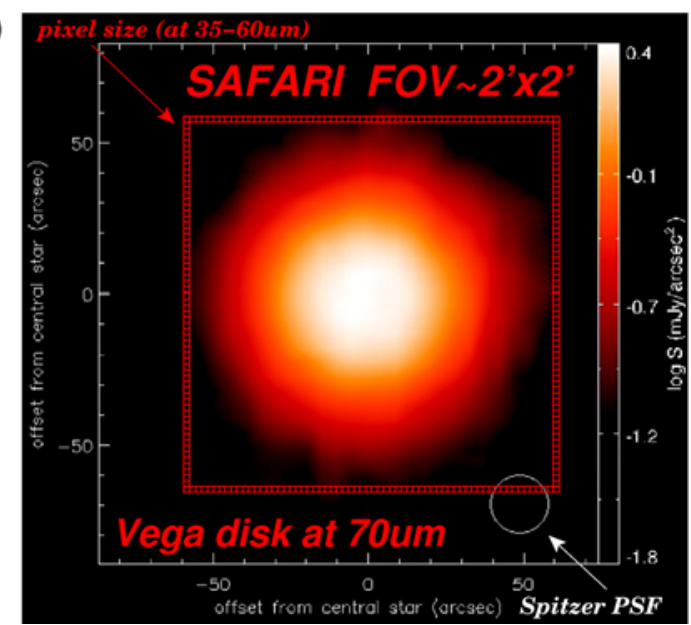

Figure 3. (a) ISO spectrum of the disk around HD142527 (Malfait et al., 1999). A these temperatures water ice can only be securely detected in the far-IR. Note that amorphous water ice only shows far-IR bands at $44 \mu m$ (Moore 8 Hudson, 1992). Such features can not be observed with Herschel or JWST. SPICA will take the equivalent spectra of objects at flux levels less than 10 mJy per minute. (c) Image of Vega debris disk at $70 \mu \mathrm{m}$ with Spitzer (Su et al., 2005). SAFARI's large, fully sampled FOV and smaller pixel size ( $\sim 1.8^{\prime \prime}$ at $\sim 44 \mu \mathrm{m}$, red squares) will provide very detailed spectro-images of nearby disks.

Taurus, Upper Sco, TW Hya, Tuc Hor, Beta Pic and Eta Cha, will provide a crucial test on the lifetime of gas and its dissipation timescales in planet forming disks and therefore on the exoplanet formation theory itself.

\subsection{Chemical COMPleXity IN Disks:}

In terms of chemistry, the protoplanetary disk is the major reservoir of key species with prebiotical relevance, such as oxygen, ammonia $\left(\mathrm{NH}_{3}\right)$, methane $\left(\mathrm{CH}_{4}\right)$ or water $\left(\mathrm{H}_{2} \mathrm{O}\right)$ to be found later in planets, asteroids and comets. But how the presence and distribution of these species relates to the formation of planets, and most particularly, rocky planets with substantial amounts of water present within the so called habitable zone, remains open to speculation without a substantial increase in observational evidence. Water is an obvious ingredient for life, and thus it is very important that we understand how water transfers from protostellar clouds and primordial protoplanetary disks to more evolved asteroids, comets and planets like our own. Ultimately one has to understand how the water we see today in our oceans was delivered to the Earth. The ability of SAFARI to detect the emission of the critical species illustrated above will produce a unique meaningful census of disk chemical composition at different evolutionary stages and shed crucial insight on the chemical evolution of the gas before and during the process of planetary formation. This in turn will tightly constrain the mechanisms by which water can be present in large quantities in the inner parts of planetary systems, i.e., the habitable zones.

\subsection{WATER ICE IN DISKS:}

Below gas temperatures of $\sim 150 \mathrm{~K}$ water vapour freezesout onto dust grains and the main form of water in the cold circumstellar disk midplane and at large disk radii will be ice. The physical location of the point at which water freezes out determines the position of the so called "snow line", i.e. the water ice sublimation front, which separates the inner disk region of terrestrial, rocky, planet formation from that of the outer giant planets (Nagasawa et al. 2007; Woitke et al. 2009a, 2009b). Grains covered by water icy mantles can play a significant role on planetary formation, enabling the formation of planetesimals and the core of gas giants protoplanets beyond the snow line. Observations of the Solar System's asteroid belt suggest that our snow line occurred near a disk radius of $\sim 2.7 \mathrm{AU}$. In the outer reaches of our own Solar system, i.e. beyond the snow line, most of the satellites and small bodies contain a significant fraction of water ice; in the case of comets this fraction is as high as $80 \%$ and the presence of water in the upper atmospheres of the four gas giants is thought to be highly influenced by cometary impacts such as that of Shoemaker-Levy 9 on Jupiter. It is possible that it is during the later phases of planetary formation that the atmospheres, and indeed the oceans, of the rocky planets were formed from water ice contained in the comets and asteroids that bombarded the inner Solar system. Disk models do predict the position of the snow line as a function of the host star type (e.g. closer to the star for cooler stars). However, very little is known from the observational point of view because the exact location of the snow line is very hard to infer with near-IR ground-based telescopes. 
In the far-IR there is a powerful tool for the detection of water ice and for the determination of the amorphous/crystalline nature: namely the transverse optical mode at $\sim 44 \mu \mathrm{m}$ both from crystalline and amorphous water ice and the longitudinal acoustic mode at $\sim 62 \mu \mathrm{m}$ arising only from crystalline water ice (Moore \& Hudson, 1992; Malfait et al., 1999). In contrast to the $\sim 3.1 \mu \mathrm{m}$ and $\sim 6.1 \mu \mathrm{m}$ water ice bands, the difference between the amorphous and crystalline phase is very well defined in the far-IR (see Fig. 3a) and, again unlike the mid-IR features, the far-IR bands are not confused with other solid state features of less abundant species in the ice (e.g., ammonia or methanol). In optically thin disks it is extremely difficult to use mid-IR absorption to trace water ice and the material is too cold to emit in the near- and mid-IR bands. Hence, these strong far-IR features are robust probes of (1) the presence/absence of water ice, even in heavily obscured or cold regions without a mid-IR background, and (2) the amorphous/crystalline state which provides clues on the formation history of water ice. Note that JWST cannot access these solid-state bands and Herschel only has access to the $\sim 62 \mu \mathrm{m}$ band with much poorer sensitivity and limited bandwidth.

First observed in the far-IR towards the Frosty Leo Nebula (Omont et al., 1990), water ice has been detected in young protoplanetary disks in a few bright sources either in the far-IR using the ISO/LWS (Dartois et al., 1998; Malfait et al., 1999) or mid-IR (Pontoppidan et al., 2005). The far-IR features were also observed using ISO/LWS in comets within our own Solar system (Lellouch et al., 1998). Since the bands change shape i.e., they narrow for crystalline ice and the peak shifts in wavelength with the temperature, relatively high spectral resolution is needed to extract all the available information from the ice band profiles. In its highest resolution mode, SAFARI provides $R \sim 4500$ at $\sim 44 \mu \mathrm{m}$ which is appropriate for very detailed ice spectroscopy studies. Indeed SPICA is the only planned mission that will allow water ice to be observed in all environments and fully explore its impact on planetary formation and evolution and the emergence of habitable planets.

\section{Concluding Remarks}

SPICA/SAFARI will detect far-IR spectral features from key chemical species $\left(\mathrm{H}_{2}, \mathrm{HD}\right.$, organic molecules, water vapor) and many bright atomic fine structure lines in protoplanetary disks. SAFARI will have enough sensitivity to detect them in a volume sufficiently large to make an unbiased study of the chemistry of protoplanetary disks out the closest star forming regions $(d \simeq 150 \mathrm{pc}$ ). The detection of the gas residual in transitional disks at the time planets assemble (at least when gas giants and "hot-Jupiters" form) will provide crucial tests to the planet formation theory itself. In the nearest (though more evolved) debris disks such as Vega or Formalhaut, SAFARI's large, fully- sampled FOV and unique access to the far-IR water ice features will allow resolving spatially the snow line (in a single fingerprint), giving a critical insight into the role of water ice in the evolution of planetary systems. For more distant disks, only a far-IR space interferometer ("FIRI"; e.g., Helmich \& Ivison 2009) can provide the angular resolution (comparable to ALMA) needed to resolve spatially the different disk layers where the emission from the above gas and ice species arises. Although FIRI will be strictly needed in the future to fully understand the formation and evolution of planetary systems, FIRI is still a long-term project. Waiting for FIRI, SAFARI will be able to observe the critical far-IR range not covered by JWST and ALMA (at comparable sensitivities) in the next decade, and with enough spectral and angular resolution to provide unique contributions to the study of protoplanetary systems.

\section{ACKNOWLEDGEMENTS}

We warmly thank the European and Japanese SPICA science teams for fruitful discussions and different contributions to develop the "protoplanetary disks" case for SAFARI. JRG is supported by a Ramón y Cajal research contract.

\section{REFERENCES}

Agúndez, M. et al. 2008, A\&A, 483, 831

Bergin, E. A., et al. 2007, Protostars and Planets V, 751

Bitner, M.A. et al. 2008, ApJ, 688, 1326

Boss, A.P. 2008, ApJ, 677, 607

Carr, J.S. \& Najita, J.R. 2008, Science, 319, 1504

Cernicharo, J. et al. 2009, $A p J$, in press, arXiv:0909.0094.

Dartois, E. et al. 1998, $A \mathscr{G} A, 338, \mathrm{~L} 21$

Dartois, E. et al. 2004, $A \mathscr{\mho} A, 423,549$

Dutrey, A. et al. 2007, Protostars and Planets V, 495

Duvert, G. et al. 2000, $A \mathscr{E} A, 355,165$

Geers, V.C. et al. 2008, $A \mathscr{E} A, 459,545$

Goicoechea, J.R. et al. 2004, ApJ, 600, 214

Goicoechea, J.R. et al. 2009, ApJ, 699, L165

Gorti, U., Hollenbach, D., 2004, ApJ, 613, 424

Gorti, U., Hollenbach, D., 2008, ApJ, 683, 287

Habart, E. et al. 2006, $A \& A A, 449,1067$

Haisch, Jr. et al. 2001, ApJ, 553, L153

Helmich, F.P. \& Ivison, R. J. 2009, ExA, 23, 245

Malfait, K. et al. 1999, $A \& A, 345,181$

Meyer, M.R. et al. 2008, ApJ, 673, L181

Moore, M.H., Hudson, R.L. 1992, ApJ, 401, 353

Nagasawa, M. et al. 2007, Protostars and Planets V, 639

Najita, J.R., et al. 2007, Protostars and Planets V, 507

Lahuis, F. et al. 2007, ApJ, 665, 492

Lellouch, E. et al. 1998, $A \mathscr{E} A$, 339, L9

Omont, A. et al. ApJ, 355, L27

Pontoppidan, K.M. et al. 2005, ApJ, 622, 463

Qi, C. et al. 2006, ApJ, 636, L157

Salyk, C. et al. 2008, ApJ, 676, L49

$\mathrm{Su}, \mathrm{K}$. Y. L. et al. 2005, ApJ, 628, 487

Woitke, P. et al. 2009a, $A \mathscr{E} A, 501,383$

Woitke, P. et al. 2009b, $A \& A$, 501, L5 